\title{
SOME INFINITE SERIES IDENTITIES
}

\author{
KEVIN B. FORD
}

(Communicated by Dennis A. Hejhal)

\begin{abstract}
Certain infinite series are shown to satisfy simple identities between the square of the sum of the series and the sum of the squares of the terms of the series. The main tool is Ramanujan's ${ }_{1} \psi_{1}$ summation formula.
\end{abstract}

It is unusual for an infinite series of nonzero terms to have the property that $\sum a_{n}^{2}=\left(\sum a_{n}\right)^{2}$. In this note, we show how Ramanujan's ${ }_{1} \psi_{1}$ summation can be used to derive a class of infinite series identities of this sort, the simplest of which is

$$
\sum_{k=-\infty}^{\infty}\left(\frac{(c / d)^{k}}{1-d^{2} c^{2 k}}\right)^{2}=\left(\sum_{k=-\infty}^{\infty} \frac{(c / d)^{k}}{1-d^{2} c^{2 k}}\right)^{2} \quad\left(|c|<|d|<\left|\frac{1}{c}\right|\right) .
$$

Ramanujan's famous ${ }_{1} \psi_{1}$ summation formula [3, Chapter 16, Entry 17] states that, for $|b / a|<|z|<1$,

$$
\sum_{k=-\infty}^{\infty} \frac{(a)_{k}}{(b)_{k}} z^{k}=\frac{(a z)_{\infty}(q / a z)_{\infty}(q)_{\infty}(b / a)_{\infty}}{(z)_{\infty}(b / a z)_{\infty}(b)_{\infty}(q / a)_{\infty}}
$$

where

$$
(a)_{\infty}=(a ; q)_{\infty}=\prod_{k=0}^{\infty}\left(1-a q^{k}\right) \quad(|q|<1)
$$

and

$$
(a)_{n}=(a ; q)_{n}=\frac{(a)_{\infty}}{\left(a q^{n}\right)_{\infty}} \quad(n \in \mathbb{Z}) .
$$

A simple proof of (2) may be found in [2], and [1, pp. 30-34] contains a general discussion of the ${ }_{1} \psi_{1}$ formula and some of its applications.

We first specialize the parameters of (2) by setting $q=c^{2}, a=d^{2}, b=$ $d^{2} c^{2 n}$, and $z=(c / d) e^{i \theta}$, where $n$ is a positive integer and $\theta$ is real. Then

$$
\frac{(a)_{k}}{(b)_{k}}=\frac{\left(d^{2} ; c^{2}\right)_{k}}{\left(d^{2} c^{2 n} ; c^{2}\right)_{k}}=\frac{\left(d^{2} ; c^{2}\right)_{n}}{\left(d^{2} c^{2 k} ; c^{2}\right)_{n}},
$$

Received by the editors April 10, 1992.

1991 Mathematics Subject Classification. Primary 33D15.

Key words and phrases. Ramanujan ${ }_{1} \psi_{1}$ summation, infinite series, Lambert series. 
and formula (2) becomes, after incorporating the term $\left(d^{2} ; c^{2}\right)_{n}$ into the righthand side,

$$
\begin{aligned}
& \sum_{k=-\infty}^{\infty} \frac{(c / d)^{k} e^{i k \theta}}{\left(d^{2} c^{2 k} ; c^{2}\right)_{n}} \\
& \quad=\frac{\left(c d e^{i \theta} ; c^{2}\right)_{\infty}\left((c / d) e^{-i \theta} ; c^{2}\right)_{\infty}\left(c^{2} ; c^{2}\right)_{\infty}\left(c^{2 n} ; c^{2}\right)_{\infty}}{\left((c / d) e^{i \theta} ; c^{2}\right)_{\infty}\left(d c^{2 n-1} e^{-i \theta} ; c^{2}\right)_{\infty}\left(d^{2} ; c^{2}\right)_{\infty}\left(c^{2} / d^{2} ; c^{2}\right)_{\infty}}=: f(\theta)
\end{aligned}
$$

In particular, we have

$$
f(0)=\sum_{k=-\infty}^{\infty} \frac{(c / d)^{k}}{\left(d^{2} c^{2 k} ; c^{2}\right)_{n}}=\left(c d ; c^{2}\right)_{n-1} A_{n}
$$

where

Furthermore,

$$
A_{n}=\frac{\left(c^{2} ; c^{2}\right)_{\infty}\left(c^{2 n} ; c^{2}\right)_{\infty}}{\left(d^{2} ; c^{2}\right)_{\infty}\left(c / d^{2} ; c^{2}\right)_{\infty}}
$$

$$
\begin{aligned}
f(\theta) f(-\theta) & =\sum_{k, l=-\infty}^{\infty} \frac{(c / d)^{k+l} e^{i \theta(k-l)}}{\left(d^{2} c^{2 k} ; c^{2}\right)_{n}\left(d^{2} c^{2 l} ; c^{2}\right)_{n}} \\
& =A_{n}^{2} \frac{\left(c d e^{i \theta} ; c^{2}\right)_{\infty}\left(c d e^{-i \theta} ; c^{2}\right)_{\infty}}{\left(d c^{2 n-1} e^{i \theta} ; c^{2}\right)_{\infty}\left(d c^{2 n-1} e^{-i \theta} ; c^{2}\right)_{\infty}} \\
& =A_{n}^{2}\left(c d e^{i \theta} ; c^{2}\right)_{n-1}\left(c d e^{-i \theta} ; c^{2}\right)_{n-1} .
\end{aligned}
$$

Integrating both sides with respect to $\theta$ over $(0,2 \pi)$ and dividing by $2 \pi$, we find that

$$
\begin{aligned}
\sum_{k=-\infty}^{\infty}\left(\frac{(c / d)^{k}}{\left(d^{2} c^{2 k} ; c^{2}\right)_{n}}\right)^{2} & =\frac{A_{n}^{2}}{2 \pi} \int_{0}^{2 \pi}\left(c d e^{i \theta} ; c^{2}\right)_{n-1}\left(c d e^{-i \theta} ; c^{2}\right)_{n-1} d \theta \\
& =A_{n}^{2} \sum_{m=0}^{n-1} a_{n, m}^{2},
\end{aligned}
$$

where we have written $\left(c d e^{i \theta} ; c^{2}\right)_{n-1}=a_{n, 0}+a_{n, 1} e^{i \theta}+\cdots+a_{n, n-1} e^{(n-1) i \theta}$. Combining this with (3), we deduce that

$$
\left(\sum_{m=0}^{n-1} a_{n, m}\right)^{2} \sum_{k=-\infty}^{\infty}\left(\frac{(c / d)^{k}}{\left(d^{2} c^{2 k} ; c^{2}\right)_{n}}\right)^{2}=\sum_{m=0}^{n-1} a_{n, m}^{2}\left(\sum_{k=-\infty}^{\infty} \frac{(c / d)^{k}}{\left(d^{2} c^{2 k} ; c^{2}\right)_{n}}\right)^{2}
$$

provided $|c|<|d|<\left|c^{1-2 n}\right|$. Identity (1) then follows by setting $n=1$ and noting that $a_{1,0}=1$.

\section{REFERENCES}

1. B. C. Berndt, Ramanujan's notebooks, Part III, Springer-Verlag, New York, 1991.

2. M. E. H. Ismail, A simple proof of Ramanujan's ${ }_{1} \psi_{1}$ sum, Proc. Amer. Math. Soc. 63 (1977), 185-186.

3. S. Ramanujan, Notebooks (2 volumes), Tata Institute of Fundamental Research, Bombay, 1957.

Department of Mathematics, University of Illinois at Urbana-Champaign, Urbana, ILLINOIS 61801

E-mail address: ford@symcom.math.uiuc.edu 